\title{
Stability predictions for high-order modulators based on quasilinear modeling
}

\author{
Risbo, Lars
}

Published in:

Proceedings of the IEEE International Symposium on Circuits and Systems

Link to article, DOI:

10.1109/ISCAS.1994.409382

Publication date:

1994

Document Version

Publisher's PDF, also known as Version of record

Link back to DTU Orbit

Citation (APA):

Risbo, L. (1994). Stability predictions for high-order modulators based on quasilinear modeling. In Proceedings of the IEEE International Symposium on Circuits and Systems (Vol. Volume 5, pp. 361-364). IEEE. https://doi.org/10.1109/ISCAS.1994.409382

\section{General rights}

Copyright and moral rights for the publications made accessible in the public portal are retained by the authors and/or other copyright owners and it is a condition of accessing publications that users recognise and abide by the legal requirements associated with these rights.

- Users may download and print one copy of any publication from the public portal for the purpose of private study or research.

- You may not further distribute the material or use it for any profit-making activity or commercial gain

- You may freely distribute the URL identifying the publication in the public portal 


\title{
Stability Predictions for High-Order $\Sigma-\Delta$ Modulators Based on Quasilinear Modeling
}

\author{
Lars Risbo \\ Electronics Institute, Technical Univ. of Denmark, \\ Bldg. 349, DK-2800 Lyngby, Denmark, lrisbo@eiffel.ei.dth.dk
}

\begin{abstract}
This paper introduces a novel interpretation of the instability mechanisms in high-order one-bit Sigma-Delta modulators. Furthermore, it is demonstrated how the maximum stable amplitude range can be predicted very well. The results are obtained using an extension of the well known quasilinear modeling of the one-bit quantizer. The theoretical results are veryfied by numerical simulations of a number of realistic 4 th order modulators designed by means of standard filter design tools. The results are useful for automated design and optimization of loop filters.
\end{abstract}

\section{INTRODUCTION}

High-order Sigma-Delta modulators (SDMs) have in recent years become popular in the design of high performance data converters $[3,6]$. Unfortunately, the instability found in these modulators is not yet fully understood. Typically, high-order modulators loose the stability when the input exceeds a certain amplitude limit. Linearized modeling is probably the most widely used tool for the analysis and design of Sigma-Delta modulators $[1,2,3,4,5]$. The only non-linear circuit element, i.e. the one-bit quantizer, is modeled as a linear gain and an additive noise source. This enables the use of standard linear system theory for performance predictions. However, linearized modeling has previously not provided useful understanding of the instability. The purpose of this paper is to give new interpretations of the instability mechanism based on previous results about linearized $\Sigma-\Delta$ modeling $[1,2,3,5]$.

Section 2, 3 and 4 review the most important results of using quasilinear modeling. Novel conclusions are presented in Section $\mathbf{5}$ and the theoretical work is validated by simulations in Section 6.

\section{SDM USING A LINEARIZED QUANTIZER MODEL}

Figure 1 shows a generic SDM which includes most single quantizer modulator structures. The modulator shown consists of a one-bit quantizer, an input filter $\mathrm{G}(\mathrm{z})$, and a feedback filter $H(z)$, having a delay of at least one sample interval, i.e., the impulse response has the property: $h(k)=0$ for $k<1$. Typically, $G(z)=H(z)$ for the traditional SDM. Throughout this paper, the operation of the quantizer will be modeled as an amplification by the factor $K$ followed by the addition of a stochastic noise source, $n(k)$ (see Figure 1). Using this model, the SDM becomes a linear system with one output $p(k)$, and two inputs, i.e. the input signal $x(k)$ and the quantization noise $n(k)$. Consequently, the system can be characterized fully by two transfer functions, i.e., the Signal Transfer Function $\operatorname{STF}(z)$, and the Noise Transfer Function NTF(z):

$$
\begin{gathered}
P(z)=S T F(z) X(z)+N T F(z) N(z), \\
N T F(z)=\frac{1}{1+K \cdot H(z)} \\
S T F(z)=\frac{K \cdot G(z)}{1+K \cdot H(z)}
\end{gathered}
$$

Typical designs yield a $\operatorname{STF}(z)$ which is flat at base-band frequencies. Some designs have input filters $G(z)$ different from $H(z)$ in order to obtain a certain characteristic of $S T F(z)$ [6]. The NTF(z) should be of the high-pass type giving good base-band suppression of the quantization noise.

Generally, the linearized system will only be stable (i.e., pole moduli less than unity) for $K$-values belonging to a certain interval, i.e., $K \in] K_{\min }, K_{\max }[$. The stable $K$-interval can easily be found using Nyquist plots $[1,3,4]$.

Since typical modulator input will be heavily oversampled, much useful information is obtained merely considering DC input. In the following it will be assumed that the input signal $x(k)$ is a DC-komponent which causes the mean value of the modulator output to be equal to the parameter $m_{p}$, i.e. $\mathrm{E}\{p(k)\}=m_{p}$. Since the output signal $p(k)$ only takes the values 1 or -1 , the output signal variance can be found as follows:

$$
V\{p(k)\}=E\left\{p^{2}(k)\right\}-E^{2}\{p(k)\}=1-m_{p}^{2}
$$

The output signal consists of the DC-component of amplitude $m_{p}$ and the quantization noise filtered by the noise transfer function $N T F(z)$. If the quantization noise $n(k)$ is assumed to be white with zero mean and variance $\sigma_{n}^{2}$, one obtains:

$$
V\{p(k)\}=\sigma_{n}^{2} \int_{0}^{1}\left|N T F\left(e^{j \times x}\right)\right|^{2} d f=\sigma_{n}^{2} A(K)
$$

The $A(K)$ term is the noise power amplification factor given by the ratio between the total output noise power and the quantization noise power. Using the Parseval theorem, $A(K)$ could also be determined as the two-norm of $N T F(z)$,i.e., the sum of the squared impulse response of $\operatorname{NTF}(z)$. The leading term of the NTF impulse response must be unity due to the unit sample delay of $H(z)$ [3]; consequently, $A(K) \geq 1$.

Combining (2) and (3) one obtains a very important relationship implied by the white noise assumption:

$$
A(K)=\frac{1-m_{p}^{2}}{\sigma_{n}^{2}}
$$

\section{NTF-PROROTYPES}

In this section a very simple and yet efficient method for the design of the feedback filter $H(z)$ is briefly reviewed $[3,4]$. A suitable high-pass filter is designed as a prototype for the desired noise transfer function: 


$$
N T F(z)=\frac{A(z)}{B(z)}
$$

where $A(z)$ and $B(z)$ are $N$ 'th order $z$-polynomials. From (1) and (5) the feedback filter $H(z)$ can be derived:

$$
H(z)=\frac{1}{K} \frac{B(z)-A(z)}{A(z)}
$$

The prototype NTF-filter must be scaled such that the $A(z)$ and $B(z)$ polynomials have equal leading z-terms in order to ensure the unit sample delay of $H(z)$. Since the operation of one-bit SDM's with $G(z)=H(z)$ are invariant to scalings of the feedback filter $H(z)$ [3], the quantizer gain value, $K$, can abitrarily be set to unity in (6).

Simple feedback filters are obtained from $N$ 'th order differentiator type filter prototypes, i.e., $N T F(z)=A(z) / B(z)$ $=\left(1-\mathrm{z}^{-1}\right)^{\mathrm{N}}$. In Figure $2 A(K)$-curves for this class of feedback filters are plotted for $N=1,2,3$. More sophisticated designs are obtained using general high-pass prototypes with transmission zeros distributed in the stop-band $[3,4,5,6]$.

\section{ESTIMATION OF THE QUANTIZER PARAMETERS}

In this section the quantizer parameters will be estimated on the basis of the statistics of the quantizer input. According to the linearized quantizer model of Figure 1, the quantizer output can be expressed as:

$$
p(k)=m_{p}+K\left(e(k)-m_{e}\right)+n(k), \quad m_{e}=E\{e(k)\}
$$

This equation leaves one degree of freedom for the linearized quantizer model, i.e the choice of the quantizer gain, $K$. Once $K$ is determined the quantization noise, $n(k)$ will be given. A convenient criterion for the choice of $K$ could be to insure that the quantization noise $n(k)$ is uncorrelated with the quantizer input $e(k)$. The consequence is that the quantizer output of Eq. (7) is split into three orthogonal components, namely a DC-component, a quantization noise component and the amplified quantizer input. Calculation of the covariance between quantizer input and output using the orthogonality criterion and (7) yields:

$$
\begin{aligned}
\operatorname{Cov}\{e(k), p(k)\}= & E\left\{\left(e(k)-m_{e}\right)\left(p(k)-m_{p}\right)\right\} \\
= & \left.K \cdot E\left\{\left(e(k)-m_{e}\right)^{2}\right\}+E\left\{\left(e(k)-m_{e}\right) n(k)\right)\right\} \\
= & K \sigma_{e}^{2} \Rightarrow \\
& K=\frac{\operatorname{Cov}\{e(k), p(k)\}}{\sigma_{e}^{2}}
\end{aligned}
$$

It can be shown that this choice of $K$ also minimizes the quantization noise power, $\sigma_{\mathrm{n}}^{2}[1]$.

Now, the quantization noise power $\sigma_{n}^{2}$ can be found by calculating the variance of the quantizer output $p(k)$ using the othogonality and (2), (7), (8):

$$
\begin{gathered}
V\{p(k)\}=1-m_{p}^{2}=K^{2} \sigma_{e}^{2}+\sigma_{n}^{2} \Rightarrow \\
\sigma_{n}^{2}=1-m_{p}^{2}-K^{2} \sigma_{e}^{2} \\
=1-m_{p}^{2}-\left\{\frac{\operatorname{Cov}\{p(k), e(k)\}}{\sigma_{e}}\right]^{2}
\end{gathered}
$$

For a known probability density function (pdf) of the quanti- zer input $e(k)$ it is fairly easy to calculate $K$ and $\sigma_{\mathrm{n}}^{2}$ using (8) and $(9)[1,4]$. Furthermore, it can be shown that the quantization noise power $\sigma_{n}^{2}$ for one-bit quantizers does not depend on the quantizer input variance $\sigma_{\mathrm{e}}{ }^{2}$. If the quantizer input pdf is assumed to be Gaussian, the quantizer noise power, $\sigma_{n}^{2}$, is given by [1]:

$$
\sigma_{n}^{2}=1-m_{p}^{2}-\frac{2}{\pi} \exp \left(-2\left(\operatorname{erf}^{-1}\left(m_{p}\right)\right)^{2}\right)
$$

For uniform pdf, $\sigma_{\mathrm{n}}{ }^{2}$ is given by [4]:

$$
\sigma_{n}^{2}=1-m_{p}^{2}-\frac{3}{4}\left(1-m_{p}^{2}\right)^{2}
$$

An interesting result is that when the quantizer input pdf is known, the noise amplification factor $\mathrm{A}$ is given as the right hand side of (4) and $A$ depends on $m_{p}$ and the shape of the pdf only. On Figure 3 the noise amplification factor $A\left(m_{p}\right)$ is plotted for both Gaussian and uniform quantizer input pdf's. It can be seen that uniform pdf gives higher A-values than Gaussian pdf for low $m_{p}$-values. For higher $m_{p}$-values vice versa. A common property is that $A\left(m_{p}\right)$ declines to unity for unity $m_{p}$.

\section{EQUILIBRIUM AND STABILITY PREDICTION}

The main result of Section 2 was that when the quantization noise is supposed to be white, the noise amplification factor $A$ of the linearized SDM has to operate along the $A(K)$ curve given by the feedback filter (cf. (3)\&(4)). Thus, the objective is to predict a kind of equilibrium point on the $A(K)$-curve. In Section 4 it was established that the noise amplification factor $A$ found for the quantizer depends of the $m_{p}$-value and the quantizer input pdf alone. Therefore, the equilibrium point satisfies the condition $A(K)=A\left(m_{p}\right)$. Furthermore, assuming that the quantizer input is e.g. Gaussian, the $A(K)$ equilibrium point can be predicted for a given feedback filter and given $m_{p}$-value: From Figure 3 (cf. (10)\&(4)) the $A\left(m_{p}\right)$-value is found. Subsequently, the equilibrium quantizer gain $K$ can then be found graphically from the graph of $A(K)$ for the used feedback filter (cf. (3)). The equilibrium point can be perceived as stable in a statistical sense if the slope of the $A(K)$-curve is positive. The argument is as follows: if the equilibrium is perturbed slightly giving e.g. a higher $K$, the result will be a higher $A(K)$-value corresponding to a higher noise amplification. This means that more noise will circulate around the feedback loop which forces the quantizer to respond with a lower $K$. This mechanism will force the system back to the equilibrium point. If the slope of the $A(K)$ curve is negative, even small perturbations will destabilize the equilibrium.

The positive slope of the $A(K)$-curve around the stable equilibrium will shift the equilibrium point towards lower $K$ and $A$-values for higher $m_{p}$. A lower $A$-value means more quantization noise power, $\sigma_{n}^{2}$, and a lower $K$-value results in less noise suppression in the base-band. These two mechanisms imply that the base-band noise power increases with $m_{p}$ which is in agreement with simulations [6].

Generally, the $A(K)$-curve for a feedback filter has a global minimum value, $A_{\min }$, along the stable $K$-interval. No equilibrium exists at all if $A\left(m_{p}\right)$ for the quantizer is lower than $A_{\min }$ 
for the feedback filter. Consequently, the $A_{\min }$-value defines a maximum modulator output DC-amplitude, $m_{p, \max }$, for a given quantizer input pdf. The next section will show that $m_{p, \max }$ for Gaussian pdf is a very good prediction of the maximum output amplitude for stable modulator operation.

Three different types of $A(K)$ curves have been found:

Type I: $\quad A_{\min }=A(0)=1, A(K)$ is monotonously increasing.

Type II: $A_{\min }=A(0)>1, A(K)$ is monotonously increasing.

Type III: $A(K)$ is $\cup$-convex and $A(K) \rightarrow+\infty$ for both $K \rightarrow K_{\min }$ and $K \rightarrow K_{\max }$

The first order SDM has a type I $A(K)$ curve (Figure 2, $N=1$ ). This implies that this first order SDM is unconditionally stable for any $m_{p}$, as $A_{\min }=1$. This is also confirmed by practical simulations.

The typical second order SDM has a type II $A(K)$ curve $\left(\mathrm{N}=2\right.$ on Figure 2) and $A_{\min }$ is greater than unity (approx. 1.6). The consequence is that the second order SDM cannot operate with $m_{p}$-values greater than approx. 0.8 when the quantizer input is assumed to be Gaussian. This is in agreement with simulations of the real second order SDM which show that the quantizer input becomes virtually unbounded for $m_{p}$ near unity.

High-order modulators have allways type III $A(K)$-curves. This implies that the equilibrium $K$-value is ambiguous, i.e., generally two $K$-values exist having the same $A(K)$-value. Only the equilibrium point with the highest $K$-value has a positive slope. Therefore, stable operation takes place only along the branch of the $A(K)$-curve between the $A_{\min }$-point and $K_{m a x}$. One can imagine that the equilibrium point escapes along the unstable branch of the $A(K)$-curve with negative slope, when $A_{\min }$ is reached. The modulator is then locked into a state with very low quantizer gain, $K$, and oscillations with large amplitude. The type III behaviour thus explains the abrupt onset of instability which is characteristic to high-order modulators. Furthermore, the type III systems will be trapped in the unstable branch of the $A(K)$-curve even when the input signal is removed.

Figure 2 shows that $A_{\text {min }}$ is greater than 9 for the third order differentiator type $(\mathrm{N}=3)$ feedback filter and consequently, this filter is completely unstable with a one-bit quantizer. Notice also that $A_{\min }$ is taken for $K<1$. Such filters with high $A_{\min }$ are only stable with multibit quantizers which allow greater $A$-values. The lack of stability of the one-bit third order modulator based on the differentiator $N T F(z)$ prototype has possibly scared many designers from using high-order feedback filters.

In order to design stable high-order modulators, a limitation of the high frequency gain of the NTF $(z)$ prototype is necessary $[3,4,5]$. This also reduces the $A_{\min }$-value of the feedback filter [4] and thereby increases the stable amplitude range. It should be emphasized that the importance of the noise amplification factor as a design parameter has been pointed out very early in [2]; However, the influence of varying the quantizer gain was not addressed in [2].

\section{DESIGN EXAMPLE AND MODEL VALIDATION}

Five different fourth order feedback filters were designed from high-pass filter $N T F(z)$ prototypes using Eq. (6).
Standard routines from the MATLAB Signal Processing Toolbox were used. The filters were designed for 64 times oversampling. The five prototype filters share the same $N T F(z)$ numerator $A(z)$ obtained from the MATLAB commands:

$$
\begin{aligned}
& {[A, \text { dummy }]=\text { cheby }\left(4,50,1 / 64,{ }^{\prime} \text { high' }\right) ;} \\
& A=A / A(1) ;
\end{aligned}
$$

The resulting normalized $A(z)$ polynomial has two complex zero pairs on the unit circle giving good noise suppression in the base-band. The denominator polynomial, $B(z)$, is of the Butterworth type and is obtained using the command:

$B=$ butter (4,fp,'high')

Where $\mathrm{fp}$ is the corner frequency of the resulting $B(z)$ polynomial. The $B(z)$ polynomial reduces the gain of the $N T F(z)$ prototype at high frequencies and thereby reduces the $A_{\text {min }}$-value. The five feedback filters have different fp-values according to Table 1 . The variation of fp gives different $A_{\min }$ values and different noise-shaping properties. Generally, a better noise-shaping is gained at the expense of a higher $A_{\min }{ }^{-}$ value.

Simulations were performed on SDM's using the five feedback filters. The mean output values, $m_{p}$, were increased from zero to unity in steps of 0.01 . For each step, 100,000 timesteps were simulated and the quantizer parameters, $K, \sigma_{\mathrm{n}}^{2}$ and $A$ were estimated using proper time averages in eq. (8), (9) and (4). The highest stable $m_{p}$-values are listed in Table 1 along with the $m_{p, \max }$-value found from the $A_{\min }$-value of the feedback filters. It is seen from Table 1 that the predicted maximum stable amplitude ranges are in good agreement with the simulation results. Typically, the predicted maximum stable amplitude ranges, $m_{p, \max }$, are slightly conservative compared to the simulation results. However, the simulated values depend generally on the simulation conditions, e.g., the number of time-steps used.

Figure 4 shows the theoretical $A(K)$-curves for the five filters with dotted lines. The $A$ - and $K$-values found from simulations are plotted using solid lines. It is seen that the simulated values follows the stable branch of the theoretical $A(K)$-curve. This fact justifies the white noise assumption for the quantizer noise in Section 2. However, stability is lost somewhat before the $A_{\min }$-point is reached. Figure 3 shows the noise amplification versus the output amplitude $m_{p}$ obtained from simulations. It is observed that the $A$-curves obtained from simulations do not fit either of the theoretical $A$-curves for Gaussian and uniform quantizer input. As a rule of thu$\mathrm{mb}$, the quantization noise power $\sigma_{\mathrm{n}}^{2}$ for this class of feedback filters is fairly constant around 0.29 for moderate $m_{p}$-values. This means that the equilibrium point and performance can be predicted very well for a given feedback filter.

Furthermore, simulations as well as the quasilinear model indicates that the full-scale signal-to-noise ratio takes a maximum value when the maximum stable amplitude limit is in the range of 0.3 to 0.35 [4]. Modulators with higher amplitude limits suffer from a deterioated base-band noise suppression and modulators with lower amplitude limits allow too litle signal output power. In both cases the signal-to noise ratio deterioates. These results are omitted due to the space limitation but can be found in [4]. 


\section{CONCLUSIONS AND PERSPECTIVES}

It has been shown that the well known quasilinear modeling predicts the qualitative difference between the stability of first, second and high-order modulators. The key to this interpretation is the noise amplification curve for the feedback filter. Furthermore, the maximum stable output amplitude can be predicted by assuming the quantizer input to be Gaussian. It has been demonstrated by simulations that the predicted maximum amplitudes are very accurate for a class of realistic high-order feedback filters designed using standard tools. The stability predictions are very precise even though the quantizer input is obviously non-Gaussian in practice. The modeling framework also accounts for the increase in the base-band noise power for high modulator output amplitudes. It is believed that the results in this paper can be used for automated and optimized design of high-order Sigma-Delta modulators. The optimization should maximize the signal-to-noise ratio under a suitable stability constraint.

\section{REFERENCES}

[1] S. H. Ardalan and J. J. Paulos, "An analysis of nonlinear behavior in delta-sigma modulators," IEEE Trans. Circuits Sys., vol. CAS-34, pp. 593-603, June 1987.

[2] B. P. Argrawal and K. Shenoi, "Design methodology for $\Sigma \Delta \mathrm{M}, "$ IEEE Trans. Commun., vol. COM-31, pp. 360-370, Mar. 1983.

[3] R.W. Adams, P.F. Ferguson Jr., S. Vincellette, A. Ganesan, T. Volpe, B. Libert, "Theory and practical implementation of a 5th-order Sigma-Delta A/D converter," J. Audio Eng. Soc., vol. 39, pp. 515-528, July/Aug. 1991.

[4] L. Risbo, Synthesis and simulations of stable high-order Sigma-Delta modulators, Masters Thesis (in Danish), Electronics Institute, Technical University of Denmark, Aug. 1991.

[5] K. C. H. Chao, S. Nadeem, W.L. Lee and C.G. Sodini, "A Higher order topology for interpolative modulators for oversampling A/D conversion," IEEE Trans. Circuits. Sys., vol. CAS-37, pp. 309-318, Mar 1990.

[6] L. Risbo, "FPGA Based 32 Times Oversampling 8thorder Sigma-Delta Audio DAC", 96th Audio Eng. Soc. Convention, Amsterdam, The Netherlands, Feb. 1994.

\begin{tabular}{|c|c|c|c|c||}
\hline Filter\# & $\mathrm{fp}$ & $\mathrm{A}_{\min }$ & $\mathrm{m}_{\mathrm{p}, \max }$ & $\begin{array}{c}\text { sim. max. } \\
\mathrm{m}_{\mathrm{p}}\end{array}$ \\
\hline 1 & 0.08 & 1.72 & 0.71 & 0.71 \\
\hline 2 & 0.10 & 1.99 & 0.58 & 0.61 \\
\hline 3 & 0.12 & 2.29 & 0.44 & 0.47 \\
\hline 4 & 0.13 & 2.46 & 0.34 & 0.41 \\
\hline 5 & 0.14 & 2.64 & 0.21 & 0.26 \\
\hline
\end{tabular}

Table 1 Filter parameters.

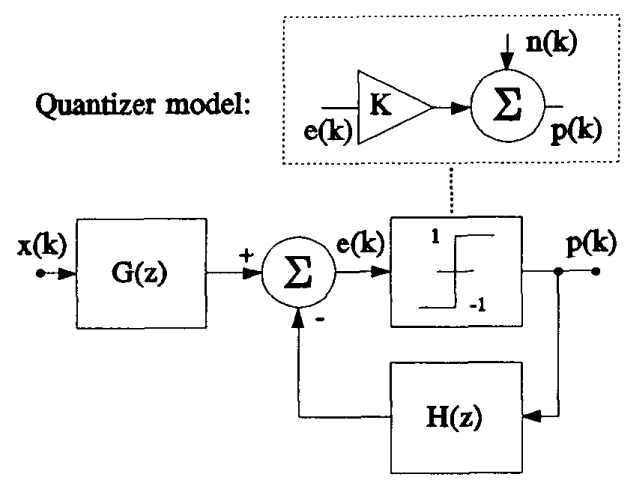

Figure 1 Generic SDM with linear quantizer model.

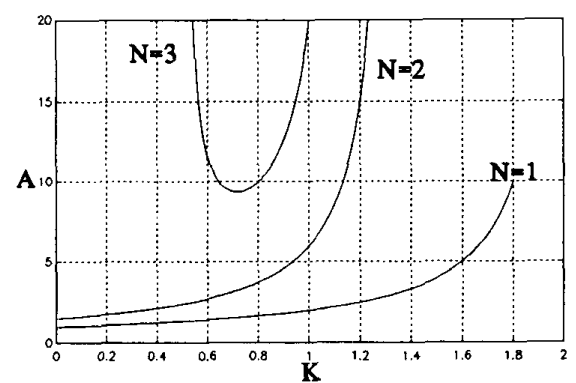

Figure $2 A(K)$-curves for feedback filters from $N^{\prime}$ th order differentiator NTF prototypes.

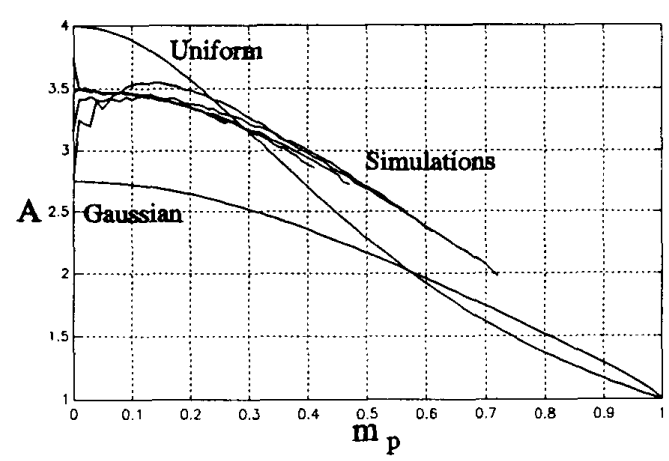

Figure $3 \mathrm{~A}$ vs. $\mathrm{m}_{\mathrm{p}}$ for the simulations and for the quantizer with Gaussian and uniform input.

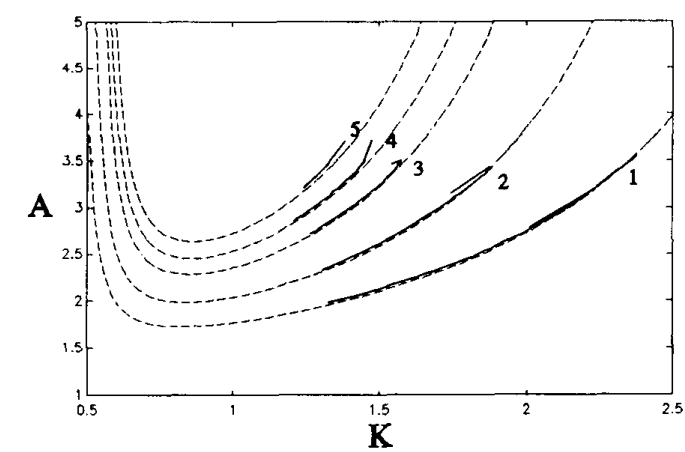

Figure 4 Theoretical and simulated $A(K)$-curves for five feedback filters. 